\title{
Tubular neighbourhoods for submersions of topological manifolds
}

\section{David B. Gauld}

\begin{abstract}
Let $\varphi: M \rightarrow N$ be a submersion from a metrizable manifold to any (topological) manifold, let $B \subset M$ be compact, $y \in N$ and $C \subset \varphi^{-1}(y)$ be a compact neighbourhood (in $\varphi^{-1}(y)$ ) of $B \cap \varphi^{-1}(y)$. It is proven that there is a neighbourhood $U$ of $y$ in $N$ and an embedding $\varepsilon: U \times C \rightarrow M$ such that $\varphi \varepsilon$ is projection on the first factor, $\varepsilon(y, x)=x$ for each $x \in C$, and $B \cap \varphi^{-1}(U) \subset \varepsilon(U \times C)$. The main application given is to topological foliations, it being shown that if $C$ is a compact regular leaf of a foliation $P$ on $M$ then every neighbourhood of $C$ contains a saturated neighbourhood which is the union of compact regular leaves of $F$.
\end{abstract}

\section{Introduction}

Throughout this paper, by an n-manifold or just manifold we will mean a topological space in which each point has a neighbourhood homeomorphic to euclidean space $R^{n}$. In particular, manifolds are not assumed to be Hausdorff. By a submersion is meant a map $\varphi: N^{m} \rightarrow N^{n}$ between manifolds, satisfying the following condition: for each $x \in M$, there are embeddings $f: R^{m}+M$ and $g: R^{n}+N$ such that $f\left(R^{m}\right)$ is a neighbourhood of $x$ in $M$ and $g^{-1} \varphi f=\varphi: R^{m} \rightarrow R^{n}$, where $p$ is projection onto the first $n$ co-ordinates. For each $y \in \varphi(M)$, the set $\varphi^{-1}(y)$, which is an

Received 19 September 1972. 
(m-n)-submanifold of $M$, is called the fibre over $y$.

Our main result is the following, the differential analogue of which has been obtained by Short and Smith [4, Lemmas 3 and 4].

THEOREM 1 (Tubular Neighbourhood Theorem). Let $\varphi: M^{m}+N^{n}$ be a submersion where $M$ is metrizable, let $y \in N$, let $B \subset M$ be compact and let $C \subset \varphi^{-1}(y)$ be a compact neighbourhood (in $\varphi^{-1}(y)$ ) of $B \cap \varphi^{-1}(y)$. Then there is a neighbourhood $O$ of $y$ in $N$ and an embedding $\varepsilon: O \times C+M$ such that:

(i) $\varphi \in$ is projection on the first factor;

(ii) $\varepsilon(y, x)=x$ for each $x \in C$;

(iii) $B \cap \varphi^{-1}(0) \subset \varepsilon(0 \times C)$.

The following definition appears in [5]. A topological space is strongly p-connected if every compact subset is contained in a compact p-comected subset.

COROLLARY 2. Every surjective submersion with strongly p-connected fibres is p-connected.

Corollary 2, which is the topological analogue of Theorem 1 of Smith [5], is proven in the same way as the proof of Smith's Theorem 1, but with the topological Tubular Neighbourhood Theorem used in place of the differential Tubular Neighbourhood Theorem of Short and Smith.

The second application of the Tubular Neighbourhood Theorem is to (topological) foliations. Let $M^{m}$ be a metrizable manifold. By a foliation $F$ of dimension $p$ on $M$ is meant a collection $\left\{U_{\alpha}, \varphi_{\alpha}\right\}_{\alpha \in A}$ where $\left\{U_{\alpha}\right\}$ is an open cover of $M$ and $\varphi_{\alpha}: U_{\alpha} \rightarrow R^{m-p}$ is a submersion satisfying the condition: for each $x \in U_{\alpha} \cap U_{B}$,

$$
\varphi_{\alpha}^{-1}\left(\varphi_{\alpha}(x)\right) \cap U_{B}=\varphi_{B}^{-1}\left(\varphi_{B}(x)\right) \cap U_{\alpha}
$$

For further definitions and elementary properties involving foliations, see [2]. If $x \in M$, let $L(x)$ denote the leaf of $F$ containing $x$. A leaf $L(x)$ is reguzar if $\forall y \in L(x), \exists\left(U_{\alpha}, \varphi_{\alpha}\right) \in F$ and embeddings 
$f: R^{m} \rightarrow U_{\alpha}, g: R^{m-p} \rightarrow R^{m-p}$ such that $f(0)=y, g^{-1} \varphi_{\alpha} f=8$ and

$$
\forall z \in f\left(R^{m}\right), L(z) \cap f\left(R^{m}\right)=\varphi_{\alpha}^{-1} \varphi_{\alpha}(z) \cap f\left(R^{m}\right)
$$

$L(x)$ is a p-submanifold of $M$. Let $M / F=\{L(x) \mid x \in M\}$. In $\S 3$ we will show that the quotient topology makes $M / F$ into an $(m-p)$-manifold and the projection $L$ becomes a submersion. A subset of $M$ is saturated if it is a union of leaves of $F$, that is, if it is of the form $L^{-1}(T)$ for some $T \subset M / F$.

THEOREM 3 (cf. Palais [3, Theorem VI, page 15]). Let $F$ be a foliation on a metrizable manifold $M$. If $C$ is a compact regular leaf of $F$ and $U$ is any neighbourhood of $C$ then there is a saturated neighbourhood $V$ of $C$ contained in $U$ such that $V$ is a union of compact regular leaves.

The following corollary follows from Theorem 3 just as does the differential analogue in Palais.

COROLLARY 4. Let $F$ be a foliation on a metrizable manifold $M$. If each leaf of $F$ is compact and regular then $M / F$ is Hausdorff.

\section{Proof of the Tubular Neighbourhood Theorem}

LEMMA 5. Let $\varphi: M^{m} \rightarrow N^{n}$ be a submersion, $C \subset M$ be compact and $y \in N$. Then we can find embeddings $f_{i}: R^{m} \rightarrow M \quad(i=1, \ldots, \tau)$ and $g: R^{n} \rightarrow N$ such that:

(i) $g(0)=y$;

(ii) $c \cap \varphi^{-1}(y) \subset \bigcup_{i=1}^{l} f_{i}\left(R^{m}\right)$;

(iii) for each $i$, we have $g^{-1} \varphi f_{i}=\varnothing: R^{m} \rightarrow R^{n}$.

Proof. Since every manifold is Fréchet, $\varphi^{-1}(y)$ is closed in $M$, so $C \cap \varphi^{-1}(y)$ is compact. Thus we can find a finite set of embeddings $f_{i}: R^{m} \rightarrow M$ and $g_{i}: R^{n}+N$, where $i=1, \ldots, l$ such that conditions 
(i), (ii) and (iii) of the statement hold but with $g$ replaced by $g_{i}$. One can then proceed as in the proof of Lemma 1 in [2] to alter the embeddings $f_{2}, \ldots, f_{2}$ to satisfy the requirements of Lemma 5 with $g=g_{1}$

We can now prove Theorem 1. Let $\varphi, y, B$ and $C$ be as in the statement of Theorem 1 . Let the embeddings $f_{i}: R^{m} \rightarrow M(i=1, \ldots, l)$ and $g: R^{n} \rightarrow N$ be as in Lemma 5. The neighbourhood 0 will be chosen so as to lie inside $g\left(R^{n}\right)$, so we may actually assume that $N=R^{n}$ and $g=1$ so that $y=0$. Let $U_{i}=f_{i}\left(R^{m}\right)$. We may assume that $\mathrm{Cl} U_{i}$ is compact. Refine the cover $\left\{U_{i}\right\}$ to an open cover $\left\{v_{i}: i=1, \ldots, 2\right\}$ of $C$ so that $\mathrm{Cl} V_{i} \subset U_{i}$. We will inductively construct open neighbourhoods $W_{i}$ of $\bigcup_{j=1}^{i}$ Cl $v_{j}$ so that $w_{i} \subset \bigcup_{j=1}^{i} U_{j}$, neighbourhoods $o_{i}$ of 0 in $R^{n}$ and embeddings $\varepsilon_{i}: O_{i} \times C_{i} \rightarrow M$, where $c_{i}=\varphi^{-1}(0) \cap w_{i}$, so that

(i) $\varphi \varepsilon_{i}$ is projection on the first factor and

(ii) $\varepsilon_{i}(0, x)=x$ for each $x \in C_{i}$.

Start of the induction. Let $W_{1}=U_{1}, O_{1}=R^{n}$ and define $\varepsilon_{1}: o_{1} \times C_{1}+M$ by $\varepsilon_{1}(t, x)=f_{1}\left(t+f_{1}^{-1}(x)\right)$ for $t \in R^{n}$ and $x \in C_{1}$. The expression $t+f_{1}^{-1}(x)$ denotes the sum of the vectors $t \in R^{n} \subset R^{m}$ and $f^{-1}(x) \in R^{m}$. Since the first $n$ co-ordinates of $f_{1}^{-1}(x)$ are all zero and $\varphi f_{1}=\varphi$, we see that $\varphi \varepsilon_{1}$ is the projection on the first factor.

Continuation of the induction. Suppose $w_{i}, o_{i}$ and $\varepsilon_{i}$ have been constructed. Choose open subsets $X, Y, S$ and $T$ of $H$ so that 


$$
\text { C1 } v_{i+1} \subset X \subset \operatorname{cl} X \subset Y \subset \operatorname{cl} Y \subset U_{i+1}
$$

and

$$
\bigcup_{j=1}^{i} \operatorname{cl} v_{j} \subset S \subset \mathrm{Cl} S \subset T \subset \mathrm{Cl} T \subset W_{i} .
$$

Define $w_{i+1}=S \cup X$. Then $w_{i+1}$ is an open neighbourhood of $i+1 \quad i+1$

$\bigcup_{j=1} \mathrm{Cl} V_{j}$ and $W_{i+1} \subset \underset{j=1}{U} U_{j}$.

Let $Z^{\prime}=\operatorname{Fr} W_{i} \cap \mathrm{Cl} Y \cap \varphi^{-1}(0)$, and $Z^{\prime \prime}=\operatorname{Fr} T \cap \mathrm{Cl} Y \cap \varphi^{-1}(0)$, where $\operatorname{Fr}$ denotes the frontier of a set. Let $Z=Z^{\prime} \cup Z^{\prime \prime}$.

The idea involved in constructing $\varepsilon_{i+1}$ is as follows: let $\varepsilon_{i+1}$ agree with $\varepsilon_{i}$ over $T$, construct $\varepsilon_{i+1}$ from $f_{i+1}$ as in starting the induction outside of $w_{i}$ and then use the isotopy extension theorem of Edwards and Kirby [1] to fill in the region between $Z^{\prime}$ and $Z^{\prime \prime}$.

For $t \in R^{n}$ sufficiently close to 0 we can define an embedding $h_{t}: z \rightarrow \varphi^{-1}(0)$ by

$$
h_{t}(z)= \begin{cases}z & \text { if } z \in z^{\prime}, \\ f_{i+1}\left(f_{i+1}^{-1} \varepsilon_{i}(t, z)-t\right) & \text { if } z \in z^{\prime \prime} .\end{cases}
$$

For $t$ sufficiently close to $0, h_{t}$ is a proper isotopy having a proper extension to a neighbourhood of $Z$. Since $Z$ is compact and $\varphi^{-1}(0)$ is a metrizable manifold, by Corollary 1.2 of [1], there is an isotopy $H_{t}: \varphi^{-1}(0) \rightarrow \varphi^{-1}(0)$ such that $H_{0}=1$ and $h_{t}=H_{t} \mid z$ for all $t$ near 0 . Actually Corollary 1.2 of [1] concerns isotopies parametrized by $I$, but one can easily generalise it to the case of isotopies parametrized by a neighbourhood of 0 in $R^{n}$.

Letting $o_{i+1}$ be a suitable neighbourhood of 0 in $R^{n}$, we can define the embedding $\varepsilon_{i+1}: o_{i+1} \times C_{i+1}+M$ by 


$$
\varepsilon_{i+1}(t, x)= \begin{cases}\varepsilon_{i}(t, x) & \text { if } x \in \mathrm{Cl} T, \\ f_{i+1}\left(t+f_{i+1}^{-1} H_{t}(x)\right) & \text { if } x \in \mathrm{Cl} w_{i} \cap \mathrm{Cl} X-T, \\ f_{i+1}\left(t+f_{i+1}^{-1}(x)\right) & \text { if } x \in \mathrm{Cl} X-w_{i} .\end{cases}
$$

This completes the induction.

By restriction of the embedding $\varepsilon_{l}$, we now have a neighbourhood $o_{\eta}$ of 0 in $R^{n}$ and an embedding $\varepsilon^{\prime}: O_{Z} \times C+M$ such that

(i) $\varphi \varepsilon^{\prime}$ is projection on the first factor;

(ii) $\varepsilon^{\prime}(0, x)=x$ for each $x \in C$.

To ensure that condition (iii) in the statement of Theorem 1 is also satisfied, it may be necessary, as in Lemma 4 of [4], to take a neighbourhood 0 of 0 in $R^{n}$ which is smaller than the neighbourhood $O_{\eta}$ and then let $\varepsilon=\varepsilon^{\prime} \mid O \times C$.

\section{Foliations}

The following notation is fixed in this section. $N^{m}$ is a metrizable manifold, $F=\left\{U_{\alpha}, \varphi_{\alpha}\right\}_{\alpha \in A}$ is a follation of dimension $p$ on $M$, $N=M / F$, and $L: M \rightarrow N$ is the projection.

THEOREM 6. If all leaves of $F$ are regular, then $N$ is a manifold of dimension $m-p$ and $L$ is a submersion.

Proof. Let $x \in M$. We must find an embedding $h: R^{m-p} \rightarrow N$ so that $h\left(R^{m-p}\right)$ is a neighbourhood of $L(x)$. Use regularity to find $U_{\alpha}$ with $x \in U_{\alpha}$ and embeddings $f: R^{m}+U_{\alpha}$ and $g: R^{m-p} \rightarrow R^{m-p}$ such that $f(0)=x, g^{-1} \varphi_{\alpha} f=\beta: R^{m} \rightarrow R^{m-p}$, and

$$
\forall y \in f\left(R^{m}\right), L(y) \cap f\left(R^{m}\right)=\varphi_{\alpha}^{-1} \varphi_{\alpha}(y) \cap f\left(R^{m}\right)
$$


Define $h: R^{m-p} \rightarrow N$ by $h(t)=L f(t)$ for $t \in R^{m-p} \subset R^{m}$.

(i) $L(x) \in h\left(R^{m-p}\right)$ since $h(0)=L f(0)=L(x)$.

(ii) $h$ is continuous, being a composition of continuous functions.

(iii) $h$ is injective, for if $s, t \in R^{m-p}$ and $h(s)=h(t)$ then $L f(\varepsilon)=L f(t)$, so that $g^{-1} \varphi_{\alpha} f(\varepsilon)=g^{-1} \varphi_{\alpha} f(t)$. But $g^{-1} \varphi_{\alpha} f=\varnothing: R^{m} \rightarrow k^{m-p}$, so $s$ and $t$ must agree on the first $(m-p)$ co-ordinates, that is, $s=t$.

(iv) $h$ is an embedding. Although this is not necessarily the case, one can arrange for it by restricting $h$ to a subset of $k^{m-p}$ if necessary and then appealing to (ii) and (iii).

(v) $h\left(R^{m-p}\right)$ is open in $N$. To prove this, it suffices to show that $L^{-I} h\left(R^{m-p}\right)$ is open in $M$, which we now proceed to do.

Suppose $y \in L^{-1} h\left(R^{m-p}\right)$. Then for some $t \in R^{m-p}$, we have $L(y)=h(t)$. Let $w=f(t, 0)$. Then $L(y)=L(w)$. Now $L(w)$ is a connected, and hence path-connected, manifold. Let $\pi: I \rightarrow L(w)$ be a path with $\pi(0)=w$ and $\pi(1)=y$.

Let $\left\{0=s_{0}<s_{1}<\ldots<s_{n}=1\right\}$ be a partition of $I$, and for each $i=1, \ldots, n$, let $\left(U_{i}, \varphi_{i}\right) \in F$ and let $f_{i}: R^{m} \rightarrow U_{i}$ and $g_{i}: R^{m-p} \rightarrow R^{m-p}$ be embeddings so that $u_{1} \subset U_{\alpha}, f_{i}(0)=\pi\left(a_{i}\right)$, $g_{i}^{-1} \varphi_{i} f_{i}=\wp: R^{m}+R^{m-p}$, and $\pi\left[s_{i-1}, s_{i}\right] \subset f_{i}\left(R^{m}\right)$.

We may assume that for each $i$ and each $z \in f_{i+1}\left(R^{m}\right)$, the set $L(z) \cap f_{i+1}\left(R^{m}\right)$ is non-empty. Indeed, proceed by induction on $i$. Given $i$, let

$$
V=\left\{(q, r) \in R^{m-p} \times R^{p} \mid \exists r^{\prime} \in R^{p} \text { with } f_{i+1}\left(q, r^{\prime}\right) \in f_{i}\left(R^{m}\right)\right\} .
$$

$V$ is open in $R^{m}$ since $f_{i}\left(R^{m}\right)$ is open in $M$. If $z \in f_{i+1}(V)$, then 
$L(z) \cap f_{i}\left(k^{m}\right) \neq \emptyset$, since if $z=f_{i+1}(q, r)$ and $r^{\prime} \in R^{p}$ is such that $f_{i+1}\left(q, r^{\prime}\right) \in f_{i}\left(k^{m}\right)$, then $L(z)=L\left(f_{i+1}\left(q, r^{\prime}\right)\right)$. Further, $f_{i+1}(V) \cap L(w)=f_{i+1}\left(R^{m}\right) \cap L(w)$, so by restricting $f_{i+1}$ to some subset of $V$ homeomorphic to $R^{m}$, we can ensure that the condition

$$
z \in f_{i+1}\left(R^{m}\right) \Rightarrow L(z) \in f_{i}\left(R^{m}\right) \neq \emptyset
$$

is satisfied. Precisely, there is some positive real number $\rho$ such that $\rho B^{m-p} \times R^{p} \subset V$. Replace $f_{i+1}$ and $g_{i+1}$ by the embeddings $f_{i+1}^{\prime}$ and $g_{i+1}^{\prime}$ given by

$$
f_{i+1}^{\prime}(q, r)=f_{i+1}\left(\frac{q}{p-|q|}, r\right) \text { for all }(q, r) \in R^{m-p} \times R^{p}
$$

and

$$
g_{i+1}^{\prime}(q)=g_{i+1}\left(\frac{q}{p-|q|}\right) \text { for all } q \in R^{m-p} \text {. }
$$

With this replacement, the induction is allowed to proceed.

Now notice that $f_{n}\left(R^{m}\right)$ is a neighbourhood of $\pi(1)=y$. Moreover, $f_{n}\left(R^{m}\right) \subset L^{-l} h\left(R^{m-p}\right)$, for if $z \in f_{n}\left(R^{m}\right)$ then $L(z) \cap f_{n-1}\left(R^{m}\right) \neq \emptyset$, so $\exists_{n-1} \in f_{n-1}\left(R^{m}\right)$ with $L(z)=L\left(z_{n-1}\right)$. Continuing thus, we obtain a sequence $z=z_{n}, z_{n-1}, \ldots, z_{1}$, with $z_{i} \in f_{i}\left(R^{m}\right)$ and $L\left(z_{i+1}\right)=L\left(z_{i}\right)$.

In particular, $z_{1} \in f_{1}\left(R^{m}\right) \subset U_{\alpha}$, so that $L\left(z_{1}\right) \in h\left(R^{m-p}\right)$, and $L(z)=L\left(z_{1}\right)$. Thus $L(z) \in h\left(R^{m-p}\right)$, that is, $z \in L^{-1} h\left(R^{m-p}\right)$.

This proves that $h\left(R^{m-p}\right)$ is open and hence completes the proof that $N$ is an $(m-p)$-manifold.

With the charts $f$ and $h$ as above, it is clear that $h^{-1} L f: k^{m} \rightarrow R^{m-p}$ is projection on the first $(m-p)$ co-ordinates, so that $L$ is a submersion. 
PROPOSITION 7. Let $P^{p}$ be a non-empty compact manifold and let $Q^{p}$ be a connected Hausdorff manifold. Then any embedding $e: P \rightarrow Q$ is a homeomorphism.

Proof. We must show that $e$ is surfective. Since $Q$ is Hausdorfe, the compact set $e(P)$ is closed in $Q$. On the other hand, $e(P)$ is also open in $Q$, for if $x \in P$ then we can find a neighbourhood $U$ of $x$ which is homeomorphic to $R^{P}$. Then $e(U)$ is a subset of the manifold $Q$ and $e(U)$ is homeomorphic to $R^{p}$. Thus by invariance of domain, $e(U)$ is a neighbourhood of $e(x)$, so $e(P)$ is open in $Q$. Since $Q$ is connected, the open and closed subset $e(P)$ must be the whole of $Q$, that is, $e$ is a homeomorphism.

LEMMA 8. Let $C$ be a compact space, let $X$ be any space, let $x \in X$ and let $U$ be an open subset of $X \times C$ containing $\{x\} \times C$. Then we can find a neighbourhood 0 of $x$ in $X$ so that $O \times C \subset U$ :

The proof of this lemma is straightforward and hence omitted.

We can now prove Theorem 3. As in the proof of Theorem $I$ we can find a neighbourhood $X$ of $L(C)$ in $N$ and an embedding $\varepsilon: X \times C \rightarrow M$ so that $L \varepsilon$ is projection on the first factor and $\forall x \in C$, we have $\varepsilon(L(C), x)=x$.

$$
\varepsilon(X \times C)=L^{-1}(X) \text {. Indeed, since } L E \text { is projection on the first }
$$

factor, we have $\varepsilon(X \times C) \subset L^{-1}(X)$. Conversely, if $x \in L^{-1}(X)$, then $\varepsilon \mid\{L(x)\} \times C$ gives us an embedding of the compact manifold $C$ in the connected Hausdorff manifold $L(x)$. Thus by Proposition 7 , $x \in \varepsilon(\{L(x)\} \times C)$, and hence $\varepsilon(X \times C)=L^{-1}(X)$.

Now applying Lemma 8, we can find a neighbourhood 0 of $L(C)$ in $X$ so that

$$
L^{-1}(0)=\varepsilon(0 \times C) \subset U \text {. }
$$

Setting $V=L^{-1}(0)$, we obtain a saturated neighbourhood of the desired type. 


\section{References}

[1] Robert D. Edwards and Robion C. Kirby, "Deformations of spaces of imbeddings", Ann. of Math. (2) 93 (1971), 63-88.

[2] David B. Gauld, "Submersions and foliations of topological manifolds", Math. Chronicle 1 (1971), 139-146.

[3] Richard S. Palais, A global formulation of the Lie theory of transformation groups (Mem. Amer. Math. Soc. 22. Amer. Math. Soc., Providence, Rhode Islend, 1957).

[4] Donald R. Short and J. Wolfgang Smith, "A Vietoris-Begle theorem for submersions", Indicana Univ. Math. J. 20 (1970/71), 327-336.

[5] J. Wolfgang Smith, "Submersions with p-connected fibers", Math. $Z$. 121 (1971), 288-294.

Department of Mathematics, University of Auckland, Auck land, New Zeal and. 